\title{
Luminous and dark matter density profiles in the inner regions of a group-scale lens at $\mathrm{z}=0.6$
}

\author{
Mônica Tergolina ${ }^{\circledR}$, Cristina Furlanetto and Marina Trevisan
}

Universidade Federal do Rio Grande do Sul, Instituto de Física Av. Bento Gonçalves, 9500, 91501-970, Porto Alegre, Brazil

email: monica.tergolina@ufrgs.br

\begin{abstract}
Density profiles of galaxy groups can provide an insight on how large-scale structure in the Universe formed and evolved, since galaxy groups bridge the gap between individual galaxies and galaxy clusters. Studying the galaxy group that is gravitational lensing HELMS18, a submillimeter galaxy at $z=2.39$ from the Herschel's HerMES Large Mode Survey (HELMS), we aim to probe the total density profile by combining strong gravitational lensing with kinematics of the centrally-located galaxies and kinematics of the group members. We have high-resolution data of HELMS18 obtained with the Atacama Large Millimeter/submillimeter Array (ALMA) and multi-object spectroscopic data of the group members from Gemini-GMOS. Our main goal is to match these observations to probe the DM and stellar density profiles and to establish a complete description of this galaxy group.
\end{abstract}

Keywords. gravitational lensing: strong, galaxies: groups, galaxies: general, galaxies: highredshift

\section{Introduction}

Probing the mass distribution of structures in the Universe, from individual galaxies to galaxy clusters, is still an open question in modern Cosmology. Structure formation and evolution models can be constrained by studies of the internal mass distribution (Bartelmann et al. 2013). Of particular interest is the radial density profile of dark matter (DM), which is sensitive to baryonic processes, such as AGN and stellar feedback. As the balance of these processes vary with mass and formation history, it is essential to investigate the baryonic and DM distributions across the full mass range, from individual galaxies to clusters. Strong gravitational lensing provides a unique way to trace the mass distribution for this wide range of systems. This technique provides a measurement of the total mass inside their respective Einstein radius. Combining strong lensing (SL) with other observations enable the density profile to be measured at different radii. While baryons and DM seem to follow an isothermal density profile in early-type galaxies (ETGs), other studies found that the inner DM density profile in galaxy clusters is shallower than the Navarro-Frank-White (NFW) profile, suggesting a connection between the assembly of stars in the central galaxies and the inner halo. (Sand et al. 2008; Newman et al. 2013).

So far, very few studies focused on the measurement of the density profile in the intermediate mass range of the galaxy groups, using SL and other techniques. Thus, the main goal of this work is to probe the stellar and DM density profiles of the galaxy group that is lensing HELMS18, a dusty star-forming galaxy (DSFG) at $z=2.39$ (Nayyeri et al. 2016), by combining SL with stellar kinematics of the central galaxies and kinematics of 


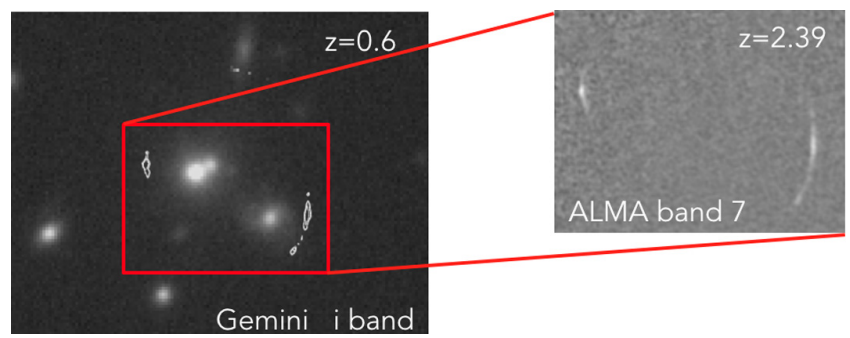

Figure 1. Superposition of Gemini and ALMA data (white contours) of the inner region of the galaxy group (a). ALMA band 7 data of HELMS18 (b).

the group members. These complementary techniques will allow us to examine the mass distribution of the group at several widely separated radii.

\section{Data and Methods}

We are using ALMA band 7 data, which show two gravitational arcs (Figure 1b). According to Amvrosiadis et al. (2018), HELMS18 system has an Einstein radius of $\sim 6.5^{\prime \prime}$. Optical images from Sloan Digital Sky Survey (SDSS) show that the lensing group has two central galaxies, an ETG and a quasar candidate (Figure 1a). Spectroscopic data from SDSS (for the ETG) indicate that the group is at $z \sim 0.6$.

We modelled the ALMA data using the semi-linear inversion method outlined in Dye et al. (2018), which works directly in the interferometric uv-plane on the visibility data. With the modelling we aim to: $i$ ) measure properties of the lens; $i i$ ) measure the slope of the density profile; $i i i$ ) reconstruct the image and investigate the properties of the background source. Preliminary results on the source reconstruction indicate a main component, which is very elongated, and a second component, located $\sim 8 \mathrm{Kpc}$ away from the main one.

As part of this project we conducted Gemini Multi-Object Spectroscopy (GMOS) observations to target both the two brightest central galaxies and the group members. Currently we are working on the data reduction. Gemini data will allow us to determine the group members, investigate stellar kinematics of the central galaxies and estimate the luminous density profile and total stellar mass. Combining SL with stellar kinematics of the centrally-located galaxies and kinematics of the group members, will allow us to measure the mass distribution of the galaxy group at different radii.

\section{References}

Amvrosiadis, A., Eales, S. A., Negrello, M., Marchetti, L., Smith, M. W. L., Bourne, N., Clements, D. L., De Zotti, G., et al. 2018, MNRAS, 475, 4939

Bartelmann, M., Limousin, M., Meneghetti, M., \& Schmidt, R. 2013, Space Science Reviews, 177,3

Dye, S., Furlanetto, C., Dunne, L., Eales, S. A., Negrello, M., Nayyeri, H., van der Werf, P. P., Serjeant, S., et al. 2018, MNRAS, 476, 4383

Nayyeri, H., Keele, M., Cooray, A., Riechers, D. A., Ivison, R. J., Harris, A. I., Frayer, D. T., Baker, A. J., et al. 2016, ApJ 823, 17

Newman, J. A., Cooper, M. C., Davis, M., Faber, S. M., Coil, A. L., Guhathakurta, P., Koo, D. C., Phillips, A. C., et al. 2013, ApJS, 208, 5

Sand, D. J., Treu, T., Ellis, R. S., Smith, G. P., \& Kneib, J.-P. 2008, ApJ, 674, 711 